\title{
Investigating Consumer Behavior towards Self- medication in the Light of Uncertainty Avoidance
}

\author{
Faryal Salman \\ SZABIST, Karachi \\ Usman Ali Warraich \\ Business Administration Department \\ Indus University, Karachi \\ Ishtiaq Ahmed \\ Bahria University, Karachi
}

\begin{abstract}
Purpose: In pursuit for seeking the understanding of the implications of Hofstede's cultural dimension, the current study assesses the relationship between uncertainty avoidance and incidence of self- medication among the consumers in Pakistan.

Methodology/Sampling: The research methodology for the study is triangulation of qualitative and quantitative techniques. Constructs were espoused from previous studies however adaptation was made to suit the researcher's needs. The instruments consisted of close ended questions only. Cross sectional data for the study has been collected through convenience sampling from a sample size of 300 students studying in different business schools in Karachi, Pakistan.

Findings: We finally conclude that the working professionals enrolled in business schools generally tend to refrain from self- medication because they consider it as risky and that it that could have serious health consequences. However it was found that people tend to self- medicate for common type of ailments based on their previous experiences. The findings also supports are assumption that high score on uncertainty avoidance means low score on behavioral intentions.

Practical Implications: The findings of the study suggest a significant relationship between behavioral intentions and uncertainty avoidance, measured through perceived risk and risk aversive behavior. The results of the study posit a negative relation between dependent and independent variables.
\end{abstract}

Keywords: Consumer Behaviors, Hofstede Model, Uncertainty Avoidance.

JEL Classification: M300, M310

\footnotetext{
* The material presented by the authors does not necessarily portray the viewpoint of the editors and the management of the Institute of Business \& Technology (IBT).

* Faryal Salman: faryal.salman@,szabist.edu.pk

* Usman Ali Warraich: Warraich.U.A@Gmail.com

* Ishtiaq Ahmed

CJMSS is published by the Institute of Business and Technology (IBT). Main Ibrahim Hydri Road, Korangi Creek, Karachi-75190, Pakistan.
} 


\section{INTRODUCTION}

In pursuit for seeking the understanding of the implications of Hofstede's cultural dimension, the current study assesses the relationship between uncertainty avoidance and incidence of selfmedication among the consumers in Pakistan. The country scores a 70 on the dimension of uncertainty avoidance, which reflects on the fact that Pakistani's tend to avoid ambiguities and uncertainties. Very thin literature is available in Pakistan to authenticate the exact statistics of self- medication practices and link it with cultural dimension. The authors of the study have attempted to explain the dimension of uncertainty avoidance through perceived risk and risk taking behavior in the light of consumer behavior towards self- medication. Self-medication is problematical not only in Pakistan but also all over the world. Such a behavior is an outcome of number of reason; reasons which encourage people to avoid consulting the physicians and resort to self- diagnosis and medication.

Nonetheless, there may be myriad motives behind opting to self- medication, more importantly we are interested in understanding the conundrums of human behavior so as to determine the factors that are pivotal in developing perceptions and attitudes towards self- medication regardless of the risks involved in such practices. Prolific contemporary literature reflects on self-medication practices in Pakistan. However there is no indigenous research that makes an attempt to understand the influence of cultural dimensions on self-medication behavior. This research is an attempt to validate Hofstede's score for Pakistan on uncertainty avoidance through risk perception towards self-medication. The findings will help marketers of high/low risk products in understanding the behavioral intentions of Pakistani consumers towards risk and avoiding ambiguities.

\section{LITERATURE REVIEW}

\subsection{Self- medication}

Self-medication which is a kind of a self-care practice has been defined as a treatment of an ailment through drugs/medicines purchased and consumed without prescription or consultation from an authorized medical practitioner (Montastrucet al., 1997). World health organization has endorsed it as an act where a person in case of suffering from some kind of sickness would succumb to self -treatment (WHO, 1998). There are many ways through which selfmedication is practiced despite the detrimental side effects. Filhoet al (2004) has identified number of reasons through which people indulge in the habit of self-medication. It can be in the form of drugs that have been used without doctor's prescription, drugs that have been used previously in similar kind of ailments, using old prescriptions to procure medicines, some previous in-stock of unused drugs at home and most importantly based on the commendation of family members, friends and co-workers. As Baig (2012) asserts that self-medication is a phenomenon that has its worldwide occurrence and it is very much part of human nature to resort to self- healing remedies.

Practice of self-medication largely based on person own comprehension of ailments and advices from a trusted source has been widely discussed (Filho et al, 2004). Global stats points high rate of its manifestation. An interesting observation here is that self-medication incidents 
are up to $68 \%$ in European countries (Bretagne et al, 2006).Nevertheless, the percentage is much higher in developing countries (Shankar et al, 2002); up to 92\% in Kuwait (Abahussain et al, 2005), 31\% in India (Deshpande \& Tiwari, 1997) and Nepal has been reported at 59\% (Shankar et al, 2002).

\subsection{Consumer Behavior Imperatives towards Self-medication}

Consumers normally indulge in self-care medication through using over the counter (OTC) sold drugs and in some cases medicines that are supposed to be used on prescription only such as antibiotics (Abasaeed et al, 2009; Sarhroodi et al, 2010). Over the counter drugs are sold without a medical prescription and are easily available at local stores and pharmacy. These drugs have a high efficacy but less side effects and are used to treat common ailments such as flu, cough and cold (Babu, 2008). Consumers normally resort to using OTC drugs due to couple of reasons such as it is more convenient due to time constraints (as doctors have long waiting hours), minor ailments can be treated at home and in case if the government is paying the medical bills less burden on national exchequer (Porteous et al., 2005; James et al, 2006). Pharmacist (Pahuja, Singh, Rohit, Gupta, Bhasin, 2011), family members, especially parents and in some cases advertisements (Verma, Mohan and Pandey, 2010) are important source of information for drugs. A study conducted in Japan identified that younger people normally resort to self- medication because it is costly and elderly do it to avoid commuting hassle (Aoyama, Koyama, Hibino, 2012). Another reason acknowledged for increase in selfmedication practices is the ease of availability of drugs and better health management practices (Bond and Hannaford, 2003). A similar study conducted in Bangladesh found that the rudimentary causes of self- medication practices are consumer's previous experience with medicines, brand familiarity and identity, trivial side effects and preconceived notions about the efficacy and effectiveness of the drug (Babu, 2008).

As consumers become more educated and literate they are able to read labels and comprehend the brief summary in leaflets normally inserted in the medicine packs. Cornucopia of literature and empirical investigation reflects on the tendency of medical and non-medical students adhering to self-care drugs(Abahussain, Matowe, Nicholls, 2005;Klemenc-Ketis,Hladnikand Kersnik, 2010; Verma et al, 2010;Sontakke, Bajait, Pimpalkhute, Jaiswal and Jaiswal, 2011; Sawalha, 2008; Olayemi, Olayinka and Musa, 2010;Martins,Miranda, Mendes, Soaresand Ferreira, 2002;Pahujaet al, 2011). Demographic factors such as age, gender and income may also affect the choice of self-care drugs (Bond and Hannaford, 2003).A study was conducted on students from public school in Kuwait on the incidence of self- medication (Abahussainet $a l, 2005)$. It was found that as students matured the tendency to self- medicate increased and also differed between male and female. Parents also influenced their children and were a source of information for self-care drugs.

However contradictory findings were reported from a web based incidence study conducted on a sample of Slovenian students. It was found that female students were more cautious in using drugs without seeking consultation from doctors however the statistical findings were not very significant among both genders (Klemenc-Ketis et al, 2010). Vermaet al (2010) researched on the self -medication practices among students in North India. Although enrolled in higher education institutes, these students had scanty knowledge towards the risk associated with selfcare medicines. They normally used drugs on their own for treating minor infirmities like headache and fever, cough \& cold, and some-times to cure abdominal Infections, mouth 
ulcer \& throat infection. Students enrolled in medical colleges due to the exposure of medical training have a better knowledge about the risks involved in self- medication (Sontakke, 2011).High self-care orientation was postulated among medical and nonmedical Palestinian students. However the type of drugs used varied according to nature of illness (Sawalha, 2008). Students studying in faculty of medicine in a Nigerian Universitywere found to be using antibiotics. The usage was due to medical background, previous experience, time constraint and some even accounted clinical staff's attitude for resorting to self-care antibiotics (Olayemi et al, 2010).

People resort to self -medication regardless of their literacy level. Illiterate people are more cautious in following rules and strictly adhered to them in particular situation (Du and Knopf, 2009). Contrary to this the literate and educated had a higher tendency to self-medicate and consider OTC drugs to have fewer side effects as compared to prescription drugs. A study conducted in Germany established the fact that children who belonged to higher socio economic strata were more exposed to self- medication, in other words there was a significant relationship between the education level of mother and her practice of self- medication(OTC drugs) towards her child (Du and Knopf, 2009).Urbanites having a higher level of education, professional status may succumb to practice self -medication because they are time barred and can't wait for longer duration outside doctor's clinic (Martins,2002).

Various studies have reported high frequency of self- medication in Pakistan (Haider \& Thaver; 1995; Zafar, Syed, Waqar S, Zubairi, Vaqar, Shaikh, Yousaf, Shahid and Saleem; 2008; Sturm Pol , Smits , HellemondtMouton , Jamil, Minai, Sampers; 1997;Mumtaz, Jahangeer,Mujtaba, Zafar and Adnan, 2011; Baig, 2012). Similar findings were obtained from almost all studies. Some common reasons found were; a) successful past experience; b) minor ailments; c) long waiting time for appointments and waiting at clinics; d) advice from family members, friends or colleagues, e)commuting issues; f)exorbitant fees of consultants especially if they are a specialist ;g) illness requires immediate care. However there is one very important contributing factor to self- medication in Pakistan. Pharmacies openly and fearlessly sell drugs without prescriptions and there are no legal repercussions for such transactions (Hussain, Malik, Hameed andRiaz, 2010). Majority of pharmacists in Pakistan are not certified practitioners. But due to their business acumen prescribe drugs and consumers opt for buying drugs from the pharmacy regardless of the risk involved in it.

\subsection{Hofstede's Cultural Dimension: Uncertainty Avoidance}

Hofested (1980) proposed a framework of cultural dimensions based on the study of IBM employees across national cultures. Through his seminal work in culture and consumer behavior Hofested found that cultural orientation varies across the globe and these dimensions have erratic effects on individual behavior (Hofested and Hofested, 2010). Hofested has identified five cultural dimensions that are prevalent in any society; a) individualism vs. collectivism; b) masculinity vs. femininity; c) power distance; d) uncertainty avoidance and e) long term orientation. The present study contemplates on the significance of uncertainty avoidance on self-medication.

Uncertainty avoidance (UAI) takes into account the risk taking behavior and tolerance for ambiguity in a society. Some societies consider obscurity as a threat and are abrasive towards 
vagueness and uncertainty. Countries that have a high score on uncertainty avoidance are bigoted towards unconventional ideas and behavior. Pakistan with a high score on UAI is considered as a risk aversive country. Uncertainty avoidance among Pakistani consumer is measured through perceived risk and risk aversive behavior towards self- medication.

\subsection{Perception of Risk in Self- medication}

The literature review of the current study reflects on some very important findings. Consumers all across the globe regardless of their education, age, gender and socio economic class resort to the act of self-medication. Although they are aware of the health risks involved in using selfcare drugs, nonetheless people resort to it because of plethora of reasons. A study had been conducted earlier in Pakistan where by it was found that student's perception of risk and hazard towards self -medication was trivial (Mumtazet al., 2006). In case of non -responsible selfmedication there are number of potential risk that consumers should be aware of such as incorrect diagnosis, delay in treatment, adverse reaction, incorrect drug administration or dosage (Ruiz, 2010). Another research was conducted by Australian Council for Safety and Quality in Health Care. The study postulated that the specific consumer behavior of using drugs referred by others or prescribed to a person suffering from similar ailment has potential hazards and risks. It might lead to using wrong drugs leading to serious health risk (Runcimanet al., 2003). This behavior of consumer has been defined as Adverse drug events(ADEs) which happens when patients use the drugs prescribed for others and in some cases share the left over drugs (Ellis, 2009).

\section{RESEARCH METHODOLOGY}

The study adopts the basic research approach based on mono method. Data is collected through single mode employing quantitative techniques. The research is explanatory study established on causal design. The theoretical framework was based on the relationship between behavioral intention (dependent variable) and uncertainty avoidance (independent variable) operationalized through perceived risk and risk aversive behavior. The basic premise of the study is that behavioral intentions towards self- medication are caused by perceived risk and risk averseness. Cross sectional data was collected using convenience sampling though selfadministered questionnaires. The target population for the study consisted of university students enrolled in executive MBA program and who were also working professionals. The total sample size was 350 students. Out of 350 questionnaires distributed 334 were returned. However 14 questionnaires were discarded because they were incomplete. In total 320 questionnaires were used for data analysis. Constructs for the study were espoused from previous studies however adaptation was made to suit the researcher's needs. The instrument for the study was divided into three sections. Section 'A' was based on a five point Likert scale and measured the relationship between the three variables selected for the study. First construct was 'behavioral intentions' measured through twelve items. Example of items are; a) If I am cured by a medicine through self-medication then I will not visit a doctor; $b$ ) I take advice for self-medication from the chemist; c) I use medicines based upon the references provided by my family and friends; d) I switch to another medicine on my own, if a doctor's prescription doesn't make me feel better. There were ten items for second construct 'perceived risk'. Example of items are; a) I may suffer harmful side-effects if I take an over/under dose of a medicine; b) I may suffer from severe allergic reactions from the medicine I use through selfmedication; c) Rules and regulations should be strictly implemented so that chemists sell 
medicines only through a doctor's prescription. Third concept 'risk aversive' tendency was measured with help of seven items. Sample items are; a) I ensure that I always take medicines that are prescribed by the doctor; b) When I take a medicine, I ensure that no health risks are involved; c) I don't want to be unsure about the medicines I take; d) I would be rather safe than sorry. Section 'B' consisted of two closed ended questions probed for the type of ailments for which consumers take self-medication and reasons for self-care drugs. The last section ' $\mathrm{C}$ ' consisted of consumer's demographic data investigating their age, education, and gender. Data was analyzed using SPSS through descriptive stats and regression analysis.

\section{ANALYSIS AND DISCUSSION}

Table 1

Respondents Demographics

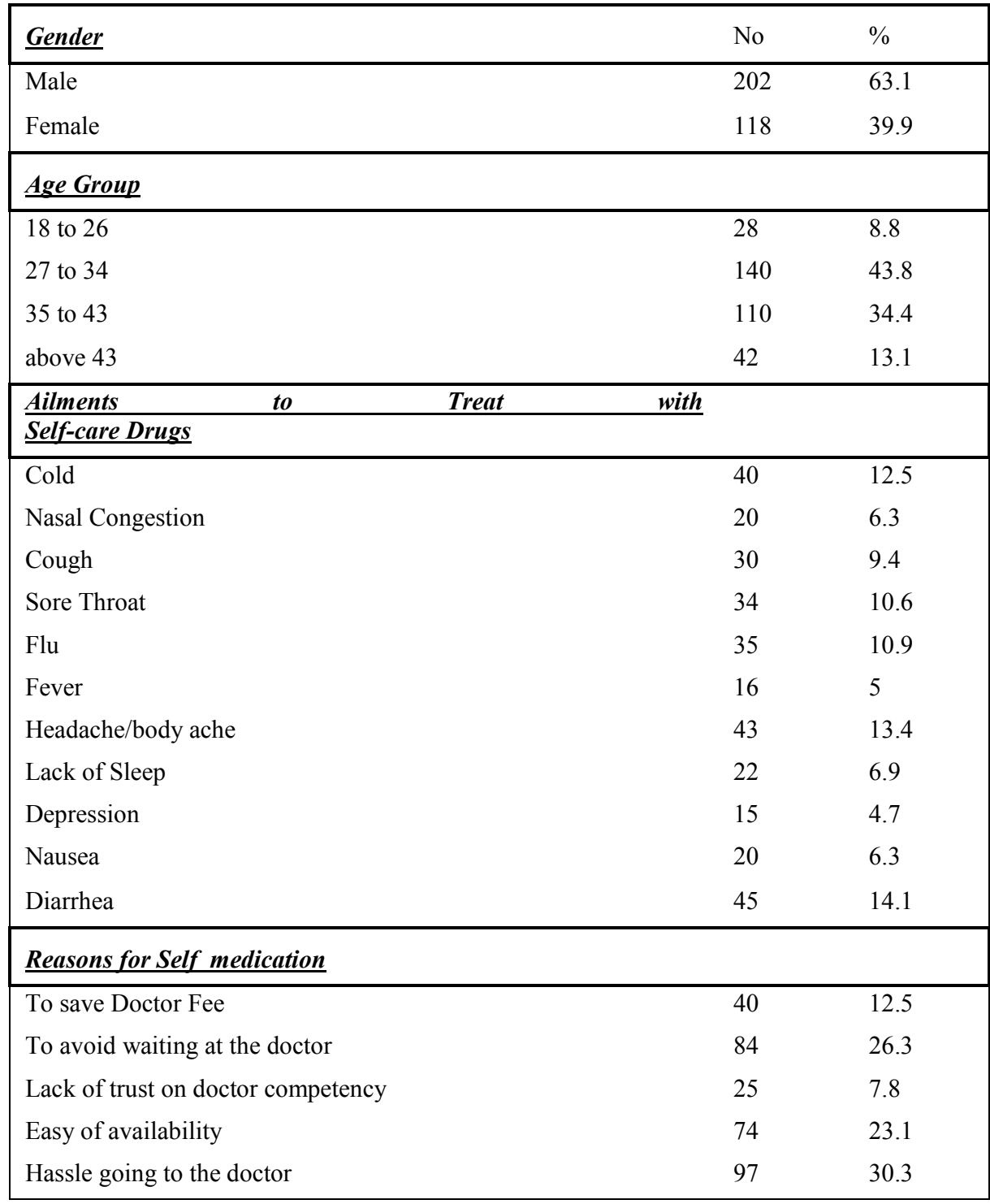

Out of 320 Respondents Male Respondents are 202 (63.1\%) and Females consists of $118(39.9 \%)$. This shows that male respondents are contributing more in this research. Further the respondents have been categorized on the basis of their age group. Out of total 320 
respondents $43 \%$ belong to the age group of 27-34 years of age (i.e. 140), below that $34.4 \%$ (110) respondents belong to the age group of 35-43 years of age and $13 \%(42)$ respondents are of more than 43 years of age. Since our target population consisted of part time students who were working professionals also therefore only $8 \%(28)$ respondents belonged to the age group of 18-26 years. This shows that the major contribution in the study belongs to respondents belonging to the age group 27-34 years.

Respondents were to identify the ailments that they tend to treat with self- care drugs. Based on past studies eleven types of illness were given as options. According to our findings consumers mostly tend to treat cold (12.5\%), Sore throat (10.6\%), Flue (10.9\%), different types of aches (13.4\%) and Diarrhea (14.1\%) with drugs without doctors prescription. Our findings are also consistentwith other studies where the similar diseases were reported to be treated with self care drugs (Babu, 2008). The findings of study points to the fact that self medication was not very common among the respondents, for Nasal congestion(6.3\%), Cough(9.4), Fever(5\%),lack of sleep(6.9),depression( $4.7 \%$ ) and nausea( $6.3 \%)$.

Respondents were asked to file reasons for self- medication. Highest number of respondents i.e. $30.3 \%$ found it much of a hassle going to the doctor. Another major reason identified was long waiting hours $(26.3 \%)$ at doctor's clinic. $74 \%$ respondents were of the view that they resort to self medication because medicines were easily available without prescription at pharmacies. $12.5 \%$ respondents used self-care drugs to save doctors fees. However lack of trust on doctors' competency was accounted as a reason by only $7 \%$.

Table 2

Behavioral Intentions

\begin{tabular}{lllll}
\hline & B & S.E & Beta & t value \\
Constant & -0.089 & 0.221 & & -0.4 \\
Perceived Risk & -.514 & .091 & -.400 & -5.681 \\
Risk Aversive & -.218 & .076 & -.202 & -2.867 \\
& & & & \\
R Square & & .095 & \\
F & & 16.733 & \\
$\mathrm{~N}$ & & 320.000 & \\
\hline
\end{tabular}

Results show that Perceived Risk and Risk aversive behavior both have significant negative relationship between Behavioral Intention. Value of $\mathrm{R}$ square shows that 9.5 percent variation in Behavioral Intention is explained by these 2 variables with significant $F$ value of 16.733 . Perceived risk has reported higher $\mathrm{t}$ value -5.681 which means that consumers perceived self medication to be risky. Nonetheless due to reasons mentioned above they resort to using drugs without doctors prescriptions. Risk averseness is negatively related to behavioral intentions which mean that consumers are cautious while using self-prescribed drugs and as our data supports that consumers use self -care drugs for only common type of ailments.

\section{CONCLUSION}


The current study is an attempt to explore consumer behavior towards self medication in the light of uncertainty avoidance. Although previous studies have contemplated on self medication practices prevalent among Pakistani consumers but they were from medical perspective. (Mumtazet al, 2011; Haider and Thaver, 1995; Zafaret al, 2008; Sturm, 1997).Very thin literature probes self-medication behavior from cultural dimension perspective. According to the findings of this study significant numbers of respondents are self -medicating but for minor diseases only. Beta for perceived risk is -.514 which strengthens Hofested score of high uncertainty avoidance in Pakistan. Consumers tend to refrain from self -medicating for illness that requires medical help such as nasal congestion, depression, lack of sleep and may involve health and safety issues. We also assume that consumers treat themselves with drugs that are easily available over the counter and do not require doctors prescription. However previous studies have also reported the effects of abuse or misuse of over the counter sold drugs (Bond and Hannaford, 2003). This information should also be communicated to consumers that OTC medicines if taken in excessive amount may have harmful side effects.

\section{FUTURE RESEARCH}

Another important cultural dimension which was not the scope of the study but greatly affects the practice of self- medication is Collectivism. Pakistan is also characterized as a highly collectivistic society. Consumer's decision making is normally influenced by reference group and other members of the clan. Our data supports this assertion that opinions and advice of family members and pharmacists also influence the habit of self -medication. Further research may deeply gage the influence of collectivism which is yet another important cultural dimension. Also the study takes a general perception of drugs. There is a need to study the difference in behavior between over the counter medicines and Prescription only medicines. There has been a major criticism on Hofetsed's work because the study had taken place almost three decades back. The consumer dynamics has changed a lot since then and Hofstede's scores on cultural dimensions may now not be validated for contemporary situation (Shariq et al., 2011). Pakistani consumer uncertainty avoidance behavior should be corroborated through conducting researches in different product categories that may not involve health and safety hazards.

\section{REFERENCES}

Abahussain, E., Matowe, L, K., \&Nicholls, P, J. (2005).Self-reported medication use among adolescents in Kuwait. Med PrincPract,14, 161-4.

Abasaeed, A., Jiri, V.,Mohammed,A., and Ales,K.(2009). Self-medication with antibiotics by the communityof Abu Dhabi Emirate, United Arab Emirates. J. Infect. Dev. Ctries, 3, 491-497. PMID: 19762966

Aoyama, I. Koyama, S.,\& Hibino,H.,(2012).Self-medication behaviors among Japanese consumers: sex, age, and SES differences and caregivers' attitudes toward their children's health management. Asia Pacific Family Medicine.11 (1), 7.doi: 10.1186/1447-056X-11-7PMCID: PMC3523005

Babu,M, M. (2008). Factors contributing to the purchase of Over The Counter (OTC) drugs in Bangladesh: An Empirical study. The Internet Journal of Third World Medicine.6 (2). DOI: $10.5580 / 1017-$ 
Bond, C., Hannaford, P. (2003) Issues Related to Monitoring the Safety of Over-The-Counter (OTC) Medicines. Dr Safe. 2003; 26:1065-1074.doi: 10.2165/00002018-200326150-00001

Du Y, Knopf H. (2009).Self-medication among children and adolescents in Germany: results of the National Health Survey for Children and Adolescents (KiGGS) Brit J Clin Pharm.,68,599-608. doi: 10.1111/j.1365-2125.2009.03477.x.

Ellis, J., Mullan, J. (2009), Prescription medication borrowing and sharing: risk factors and management. Australian Family Physician, 38(10), 2009, 816-819.

Haider, S., \& Thaver, I,H. (1995). Self- medication or self- care: implication for primary health care strategies. J Pak Med Association, 45(11), 297-8.

Hofstede, G. (1980). Culture's Consequences: International Differences in Work-Related Values., CA:Sage, Beverly Hills.

Hofstede,G., Hofstede,G,J., Minkov,M.,(2010). Cultures and Organizations: Software for the Mind. $3^{\text {rd }}$ ed.McGraw Hill.

Hussain, S., Malik, F., Hameed, A., Riaz, H. (2010). Exploring health seeking behaviour, medicine use and self medication in rural and urban Pakistan. Southern Med. Rev, 3, 32-34.

Klemenc-Ketis, Z., Hladnik, Z., and Kersnik, J.(2010).Self-medication among healthcare and nonhealthcarestudents at university of Ljubljana, Slovenia. Med. Princ. Pract, 19,395-401. DOI:10.1159/000316380

Martins, A,P., Miranda,A,C., Mendes,Z.,Soares,M,A., and Ferreira et al. (2002). Self-medication in a Portuguese urban population: A prevalence study. Pharmacoepidemiol. Drug Saf, 11, 409414. DOI: $10.1002 /$ pds.711

Mumtaz,Y., Jahangeer, S,M,A., Mujtaba,T.,Zafar, S., Adnan, S. (2011). Self Medication among University Students of Karachi, JLUMHS10, No. 03

Olayemi, O,J., Olayinka,B,O., and Musa, A,I. (2010).Evaluation of antibiotic self-medication pattern amongst undergraduate students of Ahmadu Bello university (main campus), Zaria.Res. J. Applied Sci. Eng. Techno., 2, 35-38.

Pahuja,R., Singh, H., Rohit,M., Gupta, G., Bhasin,P. (2011). An online exploratorystudy of selfmedication among pharmacy graduates in India, Int. J. Drug Dev. \& Res,3 (4), 200-207

Porteous, T., Bond,C., Hannaford,P and Sinclair,H. (2005). How and why are non-prescription analgesics used in Scotland? Fam. Pract, 22, 78-85. DOI: 10.1093/fampra/cmh719

Ruiz, ME, 2010.Risks of self-medication practices. Curr Drug Saf. 5(4):315-23.

Sarhroodi, S., A. Arzi, A.F. Swalha and A. Ashtranezhad, 2010.Antibiotic self-medication among southern Iranian university students. Int. J. Pharmacol., 6: 48-52.

Sawalha, A,F.(2008). Adescriptive study of self-medication practices among Palestinian medical and non-medical university students. Res. Soc. Adm. Pharm., 4, 164-172. DOI: 10.1016/j.sapharm.2007.04.004

Sontakke, S,D., Bajait,S.,Pimpalkhute,S,A.,Jaiswal, K,M., and Jaiswal,S,R.(2011). Comparative studyof evaluation of self-medication practices in first and third year medical students. Int. J. Biol. Med. Res,2, 561-564.

Sturm, A,W., Van der Pol R., Smits, A,J., Van Hellemondt, F,.M, Mouton, S,W., Jamil, B., Minai, A,M., Sampers, G,H. (1997).Over-the-counter availability of antimicrobial agents, selfmedication and patterns of resistance in Karachi, Pakistan,J Pak Med Association, 39(4),543-

Verma, R.K., Mohan,L., and Pandey, M. (2010). Evaluation of self medication among professional students in North India: Proper statutory drug control must be implemented. Asian J. Pharmaceut.Clin. Res, 3, 60-64. 
Wazaify, M., Shields, E., Hughes, M, C., \& McElnay, C,J.(2005). Societal perspectives on overthe-counter (OTC) medicines. FamPrac. 22,170-176.doi: 10.1093/fampra/cmh723.

Zafar, S,N., Syed, R., Waqar, S., Zubairi, A,J., Vaqar, T., Shaikh, M., Yousaf, W., Shahid, S., Saleem, S. (2008). Self-medication amongst university students of Karachi: prevalence, knowledge and attitudes. J Pak Med Association, 58(4),214-7. 